\title{
RETRACTED ARTICLE: Overexpression of thaumatin gene confers enhanced resistance to Alternaria brassicae and tolerance to salinity and drought in transgenic Brassica juncea (L.) Czern
}

\author{
Alkesh Hada ${ }^{2,3}$ - Sandhya Rawat ${ }^{1}$ - Veda Krishnan ${ }^{2,3}$ - Monica Jolly ${ }^{2}$. \\ Theboral Jeevaraj $^{3} \cdot$ Markandan Manickavasagam $^{3} \cdot$ Andy Ganapathi $^{3}$. \\ Archana Sachdev ${ }^{2} \cdot$ Anita Grover $^{1}$
}

Received: 25 March 2015 / Accepted: 4 August 2015/Published online: 20 August 2015

(C) Springer Science+Business Media Dordrecht 2015

This article has been retracted at the request of Dr. Grover, listed as corresponding author of this paper. It was found that the paper was handled by Dr. Hada instead, who submitted and approved for publication without the consent of all other authors.
The online version of this article contains the full text of the retracted article as electronic supplementary material.

Electronic supplementary material The online version of this article (doi:10.1007/s11240-015-0846-8) contains supplementary material, which is available to authorized users.

Andy Ganapathi

aganapathi2007@gmail.com

$\triangle$ Anita Grover

anita.plantbt@gmail.com

1 National Research Centre on Plant Biotechnology, New Delhi 110 012, India

2 Division of Biochemistry, Indian Agricultural Research Institute, New Delhi 110 012, India

3 Department of Biotechnology and Genetic Engineering, Bharathidasan University, Tiruchirappalli,

Tamil Nadu 620 024, India 